\title{
Finite element modeling of abradable materials - Identification of plastic parameters and issues on minimum hardness against coating's thickness
}

\author{
F. Peyraut ${ }^{1, a}$, J.-L. Seichepine ${ }^{2}$, C. Coddet $^{2}$ and M. Hertter ${ }^{3}$ \\ 1 M3M, Belfort-Montbéliard University of Technology, Belfort, France \\ 2 LERMPS, Belfort-Montbéliard University of Technology, Belfort, France \\ 3 MTU Aero Engines GmbH, Dachauer Straße 665, 80995 München, Germany
}

Received 30 October 2007, Accepted 15 February 2008

\begin{abstract}
Abradable materials are used to decrease the gas consumption of aircraft engines by minimizing the gap between the blade tips and the stator. The key idea consists in using the blades themselves to machine the gap on the abradable coating. The best compromise between soft and hard coating properties has to be reached to avoid blades wear and prevent coating erosion by gas flux and particles. The plastic parameters of abradable coating were identified by using an optimization process directly connected to FEA. The first order optimization method (conjugate gradient strategy + golden section algorithm) was applied to achieve the optimal solution. A good agreement was found between experimental and numerical results. The plastic parameters were used to study the hardness variability of abradable materials with the coating thickness. Surprisingly, a minimum hardness value was found while it was expected that hardness should be always decreasing with thickness. It has been demonstrated that this minimum is produced by the boundary conditions influence on hardness measurement. This research work was completed within the Seal-Coat project funded by the European Commission under the FP5 Growth Program.
\end{abstract}

Key words: Finite element method; optimization; HR15Y indentation test; abradable materials

\section{Introduction}

Abradable materials are used to decrease the gas consumption of aircraft engines by minimizing the gap between the blade tips and the stator [1]. These materials are located on the static parts of gas turbines, in front of blades, in order to control the overtip leakage. The key idea consists in machining the gap on an abradable coating with the blades themselves. The best compromise between soft and hard coating properties must be reached to avoid blades wear on the one hand and prevent coating erosion by gas flux and particles on the other.

In the last decades, many attempts have been made to model indentation problems with the finite element method. A detailed bibliographical review of papers published in 1997-2000 and dealing with FEM and BEM simulations of indentation problems can be found in [2]. Most of these works use general purpose FE commercial software like ABAQUS [3], ANSYS [4], MARC [5], CASTEM 2000 [6], NASTRAN [7] or ADINA [8]. To identify plastic parameters by indentation test, material properties are fitted in such a way that measured and computed hardness match. However, this fitting can be performed by various means. Cao et al. [3] have proposed to solve

\footnotetext{
${ }^{a}$ Corresponding author: francois.peyraut@utbm.fr
}

the inverse problem of identifying the plastic parameters by using an analytical dimensionless function relating the indentation response to the material properties. This approach requires preliminary computational work to fit the coefficients of the dimensionless function. Moreover, the results are only available for a restricted class of materials. To cover a wider range of alloys and pure metals, Zhao et al. [9] have extended the work of Cao and $\mathrm{Lu}$ to materials involved in engineering design, such as copper, aluminium, tin and tin alloys. Lee et al. [10] have proposed numerical formulas based on the incremental theory of plasticity for material property evaluation. The material properties used for the FE analysis cover the property range of general metals with a Young's modulus varying from 70 to $400 \mathrm{GPa}$. It does not include abradable materials for which it was found that calculated Young's moduli are closer to about 10 rather than 70 GPa. Tang and Arnell [11] have proposed a new mathematical model for the effective Young's modulus of the combined substrate and coating and the mean pressure as a measure of indentation hardness. But this model, which provides coating modulus and hardness independent on substrate effects, is only applicable for elastic-perfectly plastic materials. In [12], FEM is employed to obtain indentation parameters like maximum and residual penetration depths. 
These parameters are used in the indentation force equation which is solved by the linear regression method. However, this method only concerns materials exhibiting exponential hardening and can not be extended to others without loss of accuracy. More recently, Beghini et al. [13] have proposed an inverse procedure, starting from experimentally measured pairs of load and depth of penetration, for deducing the material parameters by means of an optimization algorithm. This procedure requires preliminary FEA to build up a database of numerical curves relating the load to the depth of penetration. Moreover, it can only be applied to plastic behaviour involving three parameters such as the Hollomon like power law or a linear strain hardening.

The limited range of applicability is generally considered as the main drawback of methods using response surface even if most classic alloys and metals are accounted. To provide a general approach applicable to any kind of material, including the abradables, an alternative is to directly connect optimization algorithms to FE analysis. Bouzakis et al. [14] have proposed this kind of approach by using FEM to extract material parameters for a multilinear plastic law. The optimization algorithm was developed by using minimization technique supported by the FEM package ANSYS. The material parameters were updated and FE computation repeated until a criterion between measured and calculated penetration forces will be fulfilled. We have selected a similar approach by using the FE code ANSYS [15]. The difference between measured and computed data was minimized to find the appropriate material properties satisfying the similarity of computational and experimental hardness. The first order optimization method (conjugate gradient strategy + golden section algorithm) was applied to achieve the optimal solution. This method is time consuming but converges accurately towards the global minimum of the objective function. Two indentation depths corresponding to two different coating thicknesses have been considered instead of the complete load-depth indentation curve related to a single coating thickness. Since two different coating thicknesses are considered for the reverse analysis, the proposed approach likely works as the dual method [16] used for sharp indentation to provide a single combination of appropriate material parameters. This ensures that the relation between material parameters and hardness is one-to-one. The proposed method can be directly applied to any kind of bilinear plastic material and does not need any preliminary computational work to fit coefficients and material constants as required by other methods.

Thanks to the identification of the plastic parameters, an extensive study of hardness against thickness was performed by using an axisymmetrical FE model of the HR15Y indentation test. Several hardness tests are available to perform hardness measurement, each one providing different hardness value (Vickers, Rockwell and Rockwell superficial, Brinell ...). Among these various hardness tests, the HR15Y indentation test [17] is the most widely used by industry manufacturers to control
Durabrade 2673 NiCrAIl5hBN flam e sprayed

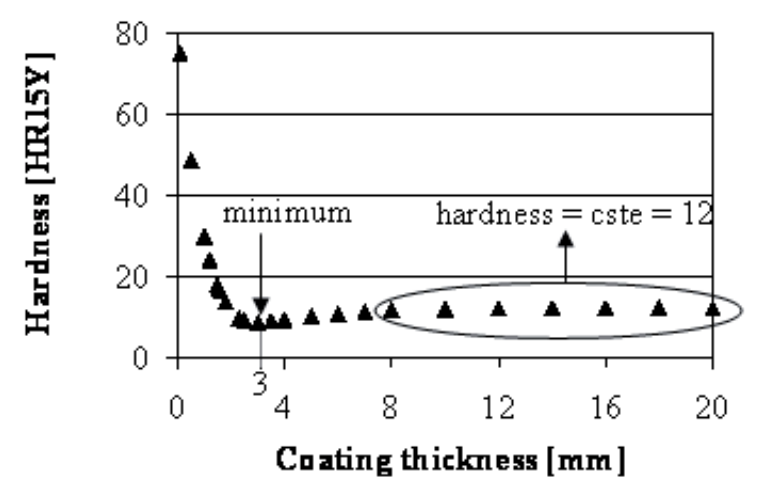

Fig. 1. Micrograph of a typical abradable coating structure; left: surface view - right: cut view.

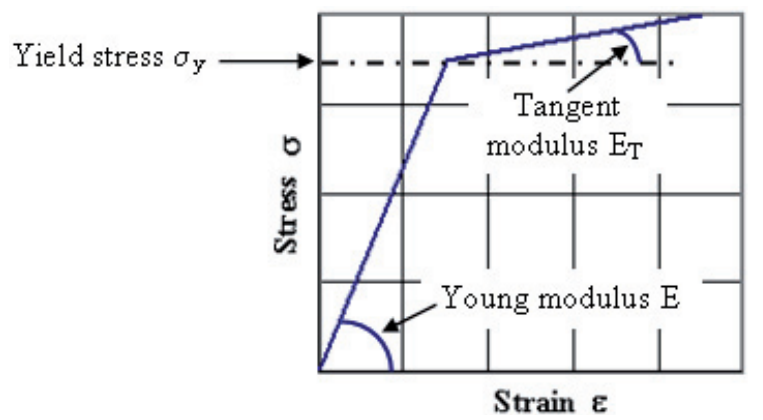

Fig. 2. Elastic-plastic behaviour law.

the quality of thermally sprayed abradable coating. The general principle is the measurement of the permanent increase in depth of penetration with a $12.70 \mathrm{~mm}$ diameter steel ball indenter, a preliminary test force of $29 \mathrm{~N}$ and a total test force of $147 \mathrm{~N}$. The FE results show a fairly good agreement with measured hardness. However, a minimum hardness value was found while it was expected that hardness should be always decreasing with thickness. By focussing the study on the central part of the coating, which is the most significant region to account for plastic effects, we have demonstrated that this minimum hardness is produced by the boundary conditions influence on hardness measurement.

\section{Problem presentation}

Abradable coatings are made of complex 3-D heterogeneous composite materials randomly distributed (Fig. 1). A bilinear plastic law was used to model the mechanical behaviour of these coatings (Fig. 2). To take into account the scale of the heterogeneities (micron) and the scale of the coating (millimetre), the elastic properties were computed thanks to a multi-scaled approach [18]. The Young's modulus and the Poisson ratio calculated with this approach for the abradable coating considered in this work are respectively $15 \mathrm{GPa}$ and 0.2 .

The plastic parameters were identified by minimizing the difference between computed and measured 


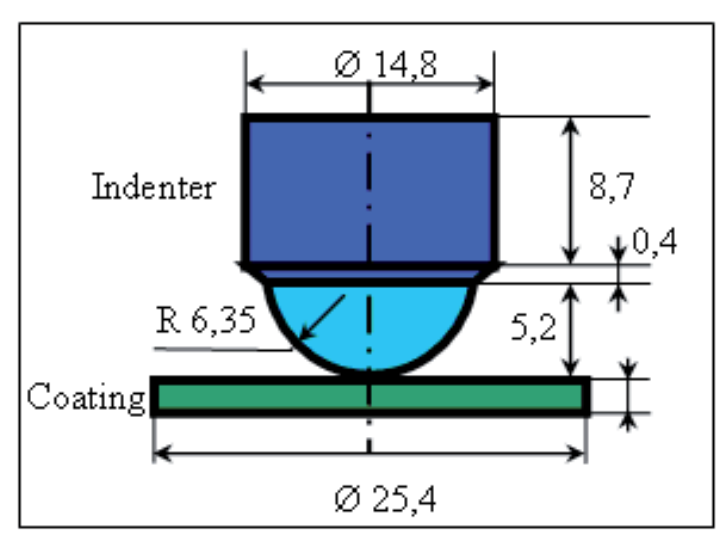

Fig. 3. Schematic of HR15Y test rig.

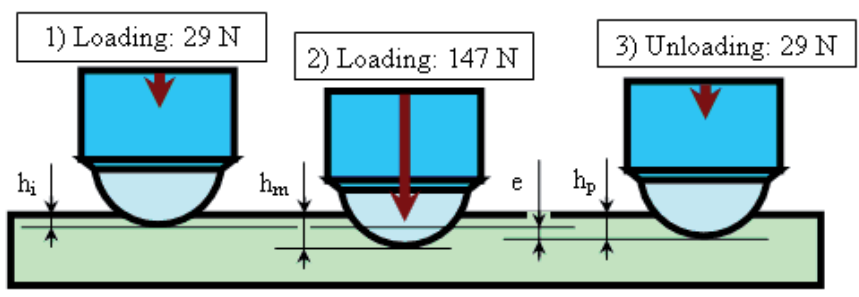

Fig. 4. Multi-step loading.

hardness's. The general principle is the measurement of the permanent increase in depth of penetration under a multi-step load. Various geometrical indenters can be used as spheroconical tip or ball with various diameters. In [19], the effects of different ball materials on HRB hardness measurement values are discussed. In our work, the standardized HR15Y test (Fig. 3) is selected because it is the most widely used by manufacturers of abradable coatings for aero-engines application. This test uses a $12.70 \mathrm{~mm}$ diameter steel ball indenter, a preliminary test force of $29 \mathrm{~N}$ and a total test force of $147 \mathrm{~N}$ (Fig. 4). The preliminary test force is applied to cause an initial penetration $h_{i}$ of the specimen in accordance with recommendations provided by hardness measurement standards [17]. The total force is next applied to provide the maximum depth of penetration $h_{m}$. The permanent depth of penetration $h_{p}$ is obtained by unloading the system from $147 \mathrm{~N}$ to $29 \mathrm{~N}$. By introducing the difference e between the permanent $h_{p}$ and the initial $h_{i}$ depths of penetration with a $\mu \mathrm{m}$ scale, the material hardness $H$ is defined as $H=100-$ e.

\section{Numerical procedures}

\subsection{Finite element model}

The FE code ANSYS was used to build a bi-dimensional model of the coating and the indenter (Fig. 5). Both of them are meshed in the $(x, y)$ plane by four nodes PLANE42 elements used with axisymmetrical option. The conventional notations used by ANSYS were adopted to represent the radius and the rotation axis (respectively $x$ and $y$ directions). The FE model is made of 2176 nodes and 2163 elements for the lowest thickness used in the present work $(0.1 \mathrm{~mm})$ and 14893 nodes and 14723 elements for the highest $(20 \mathrm{~mm})$. This model includes local non linearity to model frictionless contact between the indenter and the coating and material non linearity to represent the plastic behaviour of abradable materials. Frictionless contact is assumed because it has been observed that friction produces negligible effects on the curve relating the load to the depth of penetration [10]. TARGE169 and CONTA171 elements were used to model contact between the coating and the indenter and the mesh density is refined in the vicinity of the contact area to account for high stress gradient growing in this region. TARGE169 element represents the "target" surface and CONTA171 is a "surface-to-surface" contact element used to represent contact and sliding between 2-D "target" surfaces and a deformable surface defined by this element. The bottom of the coating was constrained against displacement in the $y$-direction and the vertical symmetry axis, defined by $x=0$, was constrained against displacement in the $x$-direction to model axisymetrical condition. To model the plastic behaviour of the coating, a bilinear isotropic law was used (Fig. 2). The material parameters are the yield stress $\sigma_{y}$, the tangent modulus $E_{T}$ and the Young modulus $E$. A preliminary FEA is performed on a $2 \mathrm{D}$ model of the material microstructure in order to compute $E$ [18]. $\sigma_{y}$ and $E_{T}$ are next identified by using a reverse procedure described in Section 3.2.

\subsection{Optimization}

The optimization process used to identify the yield stress $\sigma_{y}$ and the tangent modulus $E_{T}$ values is presented in this subsection. The idea is to determine $\sigma_{y}$ and $E_{T}$ in order to minimize the difference $D$ between measured and computed hardness

$$
\operatorname{Min}_{\sigma_{y}, E_{T}}[D] .
$$

The yield stress $\sigma_{y}$ and the tangent modulus $E_{T}$ represent the design variables and the objective function $D$ is defined by the distance between experimental and numerical hardnesses. This distance is expressed in terms of percentage by using the usual Euclidian norm

$$
D=100 \times \frac{\left\|\mathbf{H}^{c}-\mathbf{H}^{m}\right\|}{\left\|\mathbf{H}^{m}\right\|} .
$$

Measured and computed hardnesses are stored with two vectors $\mathbf{H}^{m}$ and $\mathbf{H}^{c}$

$$
\mathbf{H}^{m}=\left\{\begin{array}{l}
24.1 \\
10
\end{array}\right\} ; \mathbf{H}^{c}=\left\{\begin{array}{l}
H_{1.2}^{c} \\
H_{2.3}^{c}
\end{array}\right\} .
$$

Each component corresponds to a different thickness. Two components are needed since two plastic parameters have to be found. In order to extract the most relevant information from experimental data's, the lowest and highest coating thicknesses were logically considered (1.2 and $2.3 \mathrm{~mm}$ ). 


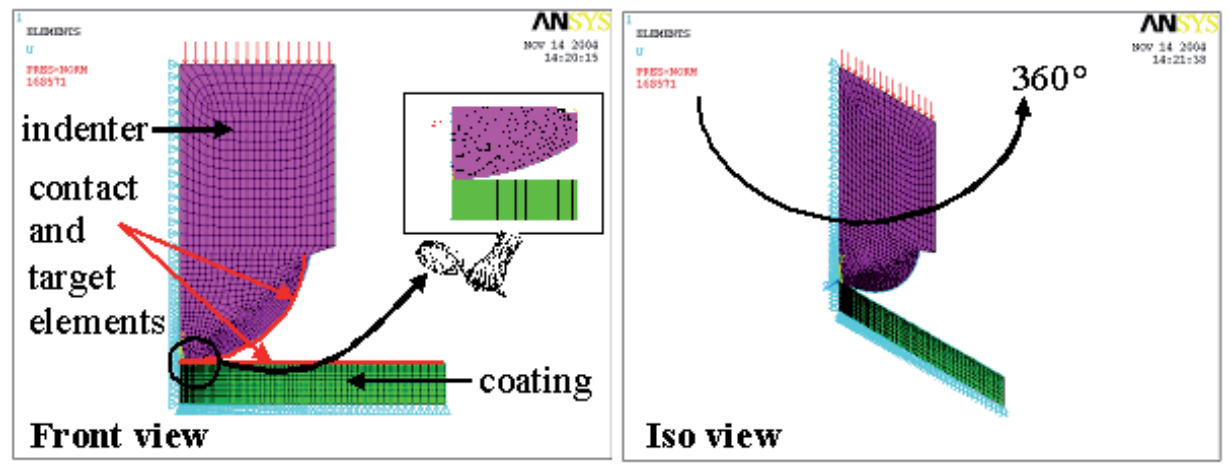

Fig. 5. Axisymmetrical finite element model.

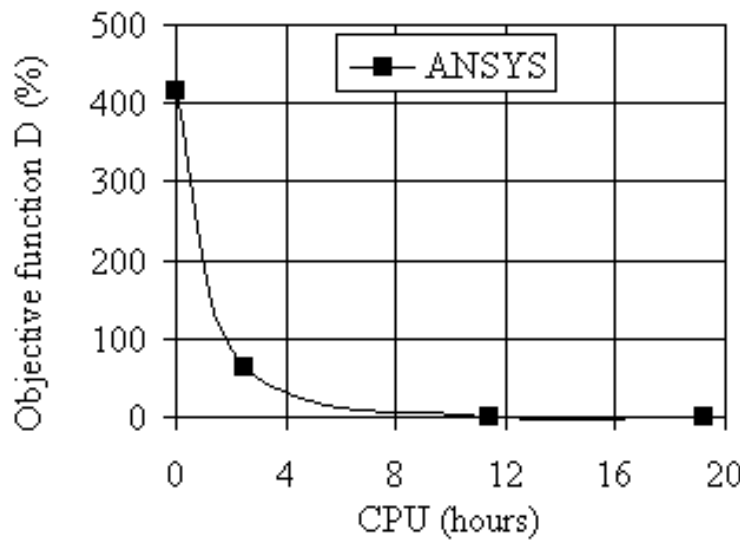

Fig. 6. Computation time.

The first order optimization method was applied to achieve the optimal solution. This method combines a conjugate gradient strategy to determine the descent direction and a golden section algorithm to perform a line search along the descent direction. It is noted in Figure 6 that nearly twenty hours are required to achieve the optimal solution on a PC (Pentium $4 / 2.8 \mathrm{GHz}$ ). The optimization procedure is thus computationally demanding. However, it is possible to reduce the computation time by about a half ( $11 \mathrm{~h}$ instead of $19 \mathrm{~h}$ ) without losing accuracy ( $D=0.86$ instead of $0.55 \%$ ). Moreover, the method is efficient and provides accurate results as it will be demonstrated in the next section.

\section{Computational results}

\subsection{Plastic parameters}

The objective function surface is depicted in Figures 7 and 8 . This surface was built by computing hardness against design variables $\left(\sigma_{y}, E_{T}\right)$ satisfying $0.5 \mathrm{Mpa} \leqslant \sigma_{y} \leqslant 10 \mathrm{Mpa}$ and $1 \% \leqslant E_{T} / E \leqslant 50 \%$. The iterative solutions provided by the optimization procedure are represented by squares. It is observed that the procedure converges perfectly well towards a minimum defined by: $\sigma_{y}=3 \mathrm{MPa}$ and $E_{T}=1.5 \% \mathrm{E}$. It is also noticed that the first iterations move from the starting

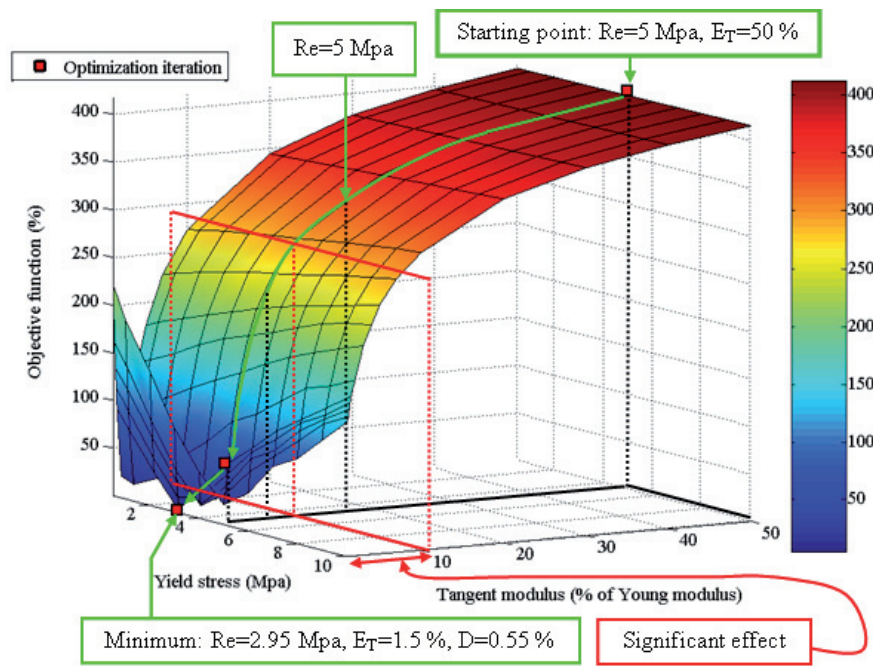

Fig. 7. Objective function versus design variables.

point along a straight line defined by $\sigma_{y}=5 \mathrm{MPa}$. The objective function is thus more sensitive to the tangent modulus than the yield stress. The most significant effect is found for $E_{T}$ changing from 0 to $10 \% E$ corresponding to the stiffest slope of the objective function. This observation is in agreement with the results obtained by Gan et al. in [20]. It is also noticed that there is only one global minimum in the considered range of parameters variation. Non-uniqueness, i.e. identical hardness's obtained from different combination of plastic parameters [21-24], is not observed. Since two thicknesses are used to perform the reverse analysis, the proposed approach likely works as the dual method [16] used for sharp indentation to provide a single combination of appropriate material parameters. It is actually noticed that two different thicknesses provide two different load-indentation depth curves (Fig. 9). This ensures that the relation between material parameters and hardness is one-to-one.

\subsection{Hardness against thickness}

The plastic parameters identified in Section 4.1 and the FE model described in Section 3.1 were used to compute hardness against coating thickness. Comparison between 


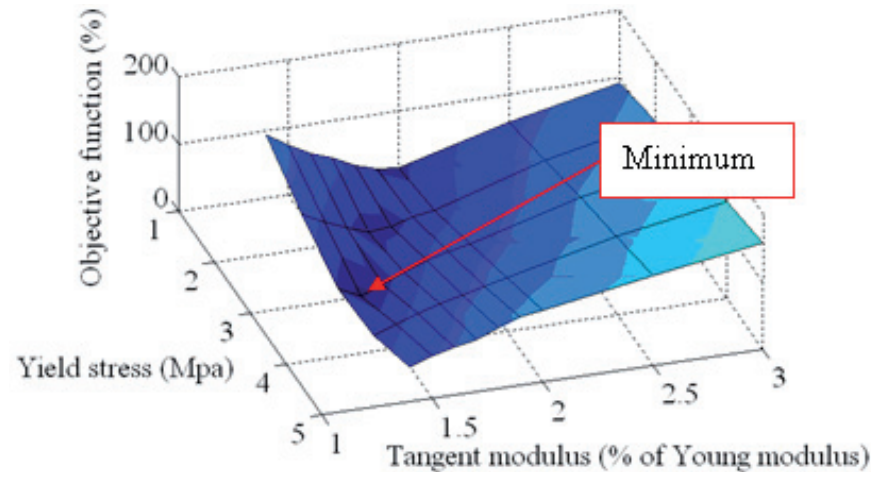

Fig. 8. Zoom on the minimum of the objective function.

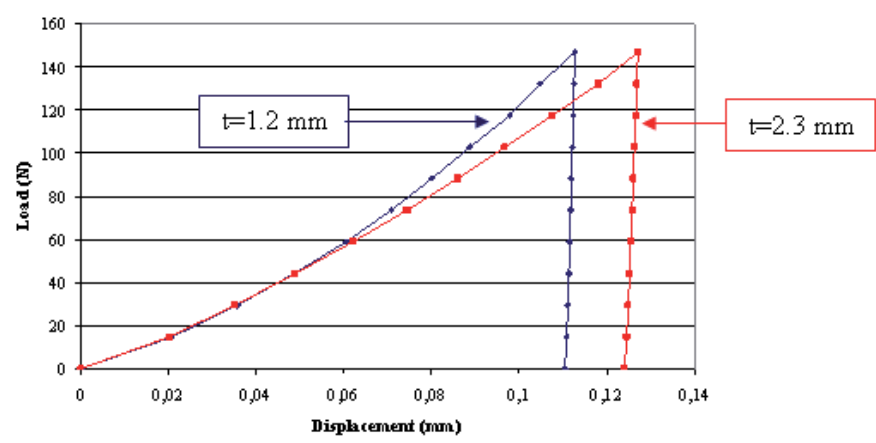

Fig. 9. Numerical load-indentation depth curves.

measured and computed hardness is presented in Figure 10. A perfect agreement is noticed if the coating thickness is equal to 1.2 or $2.3 \mathrm{~mm}$. This result was expected because these thicknesses were used to equate to zero the difference between tested and computed hardness (see Sect. 3.2). A very good agreement between numerical and experimental results is also observed for other thicknesses since the difference never exceeds $6.7 \%$ (Tab. 1). If the thickness is less than $3 \mathrm{~mm}$, it is noted that hardness decreases sharply with the thickness up to a minimum value (Fig. 11). On the contrary, if the thickness is more than $3 \mathrm{~mm}$, the hardness firstly increases and then reaches a thickness independent value. The variability of hardness against thickness has been already observed for real material and is not induced by a numerical artefact [25].

\subsection{Relationship between hardness measurements and residual stresses in the coating}

It was observed in Figure 11 that the hardness reaches a minimum for a thickness value equal to $3 \mathrm{~mm}$. By investigating the relationship between hardness and stress field, we will demonstrate in this section that the minimum hardness is produced by the boundary conditions influence on hardness measurement.

It is firstly noticed that the central part of the coating is the most significant region to account for plastic effects. The Von Mises plastic strain is actually concentrated within the range $x \in[0-2.54 \mathrm{~mm}]$ (Fig. 12). In this region, the plastic strain extends radially from the contact zone to
Durabrade 2673 NiCrAIl5hBN flame sprayed

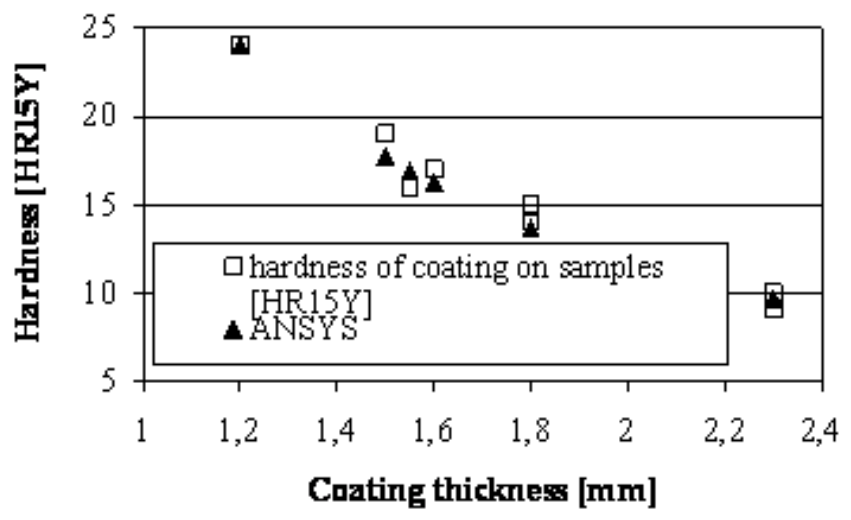

Fig. 10. Comparison between measured and computed hardness.
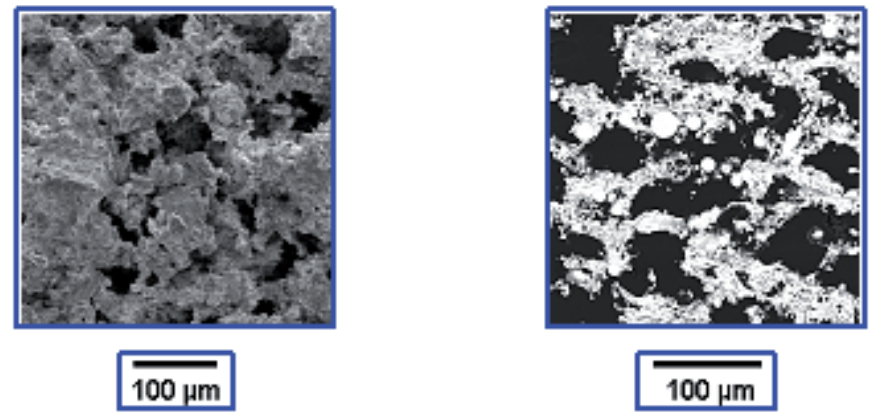

Fig. 11. Computed hardness.

the coating bottom. This general trend of radial expanding has been already observed in $[5,19,26]$. By focusing on the central part of the coating, it is noted that the coating thickness giving the maximum reaction force is equal to $3 \mathrm{~mm}$ (Fig. 13). This corresponds to the thickness value providing the minimum hardness (Fig. 11). Since the reaction force results from the constrained displacement applied to the coating bottom, this demonstrates that hardness variability, and particularly occurrence of minimum hardness, are ascribed to the boundary condition influence on hardness measurement. The distribution of the vertical stress component $\sigma_{y y}$ on the coating bottom was also studied to understand why the maximum force is related to a $3 \mathrm{~mm}$ coating thickness. Three stress curves are plotted against $x$ coordinate in Figure 14. These three stress curves are related to a $3 \mathrm{~mm}$ thickness and to two thicknesses close to $3 \mathrm{~mm}$ (2.5 and $3.5 \mathrm{~mm})$. These curves intersect each other nearly $x$ equal to $1.6 \mathrm{~mm}$. The lowest thickness provides the lowest stress value up to the intersection while the opposite trend is observed beyond the intersection. A similar conclusion was found for the reaction forces acting within the range $x \in[0,1.6 \mathrm{~mm}]$ and $x \in[1.6,2.54 \mathrm{~mm}]$ (Fig. 15). The maximum force then results from the competition between two opposite trends and depends on the stress distribution on the coating bottom. The minimal hardness observed in this work 
Table 1. Comparison between measured and computed hardness.

\begin{tabular}{cccc}
\hline Coating thickness [mm] & Measured hardness & Computed hardness & Difference (\%) \\
\hline 1,2 & 24,1 & 24 & 0,4 \\
1,5 & 19 & 17,72 & 6,7 \\
1,55 & 16 & 16,85 & $-5,3$ \\
1,6 & 17 & 16,2 & 4,7 \\
1,8 & 14,5 & 13,62 & 6,1 \\
2,3 & 9,5 & 9,6 & $-1,1$ \\
\hline
\end{tabular}

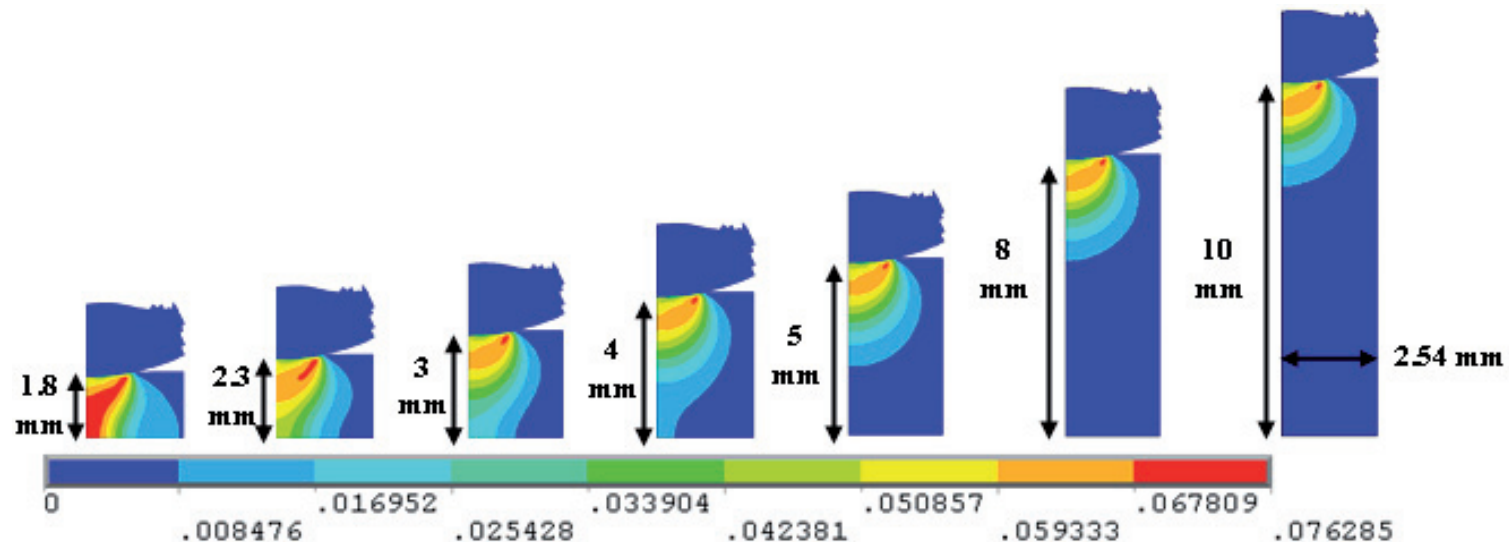

Fig. 12. Von Mises plastic strain component for various thicknesses.

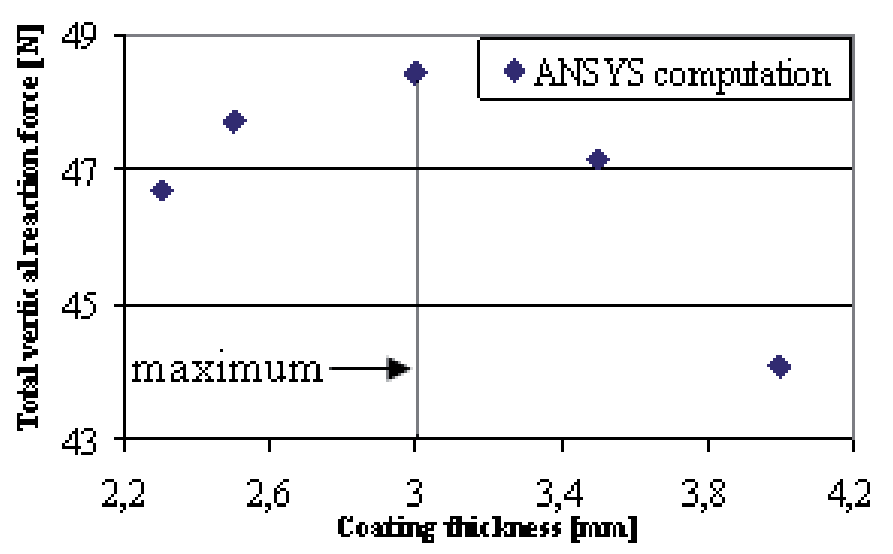

Fig. 13. Reaction force applied to the coating bottom within the range $x \in[0,2.54]$.

is thus produced by the boundary conditions influence on hardness measurement.

\section{Conclusion}

A finite element model of the HR15Y hardness test was developed to identify the plastic parameters of abradable materials. To provide a general approach applicable to any kind of material, including the abradables, the parameters identification was performed by connecting optimization algorithms to FE analysis. The first order optimization method was used in this work. This method is time consuming but has been found accurate and ef-

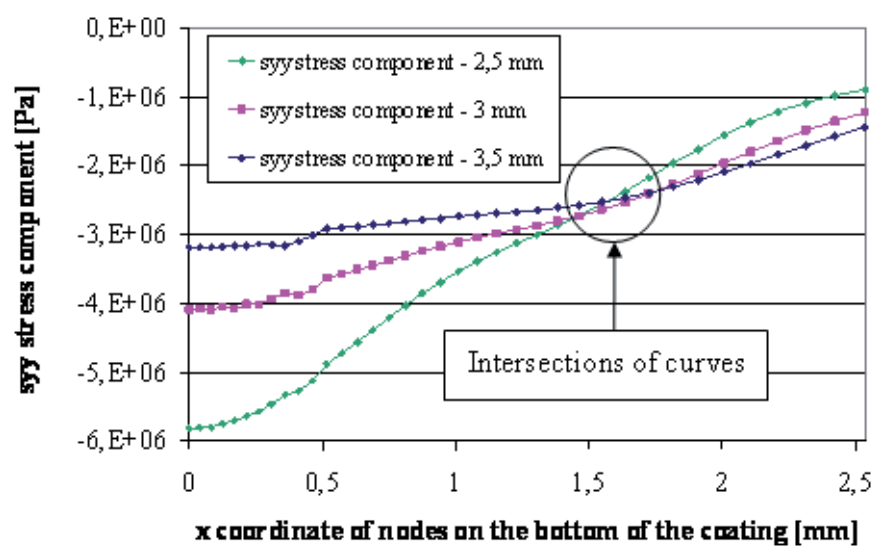

Fig. 14. $\sigma_{y y}$ stress component distributed on the central part of the bottom coating.

ficient to provide the global minimum of the objective function. In the considered range of parameters variation, non-uniqueness has not been observed (i.e. identical hardness's obtained from different combination of plastic parameters). Since two different load-indentation depth curves corresponding to two different thicknesses are considered for the reverse analysis, the proposed approach likely works as the dual method used for sharp indentation to provide a single combination of appropriate material parameters.

The plastic parameters were used to study the variation of hardness with the coating thickness. The results show a fairly good agreement between computed and measured hardness. However, a minimum hardness value was 


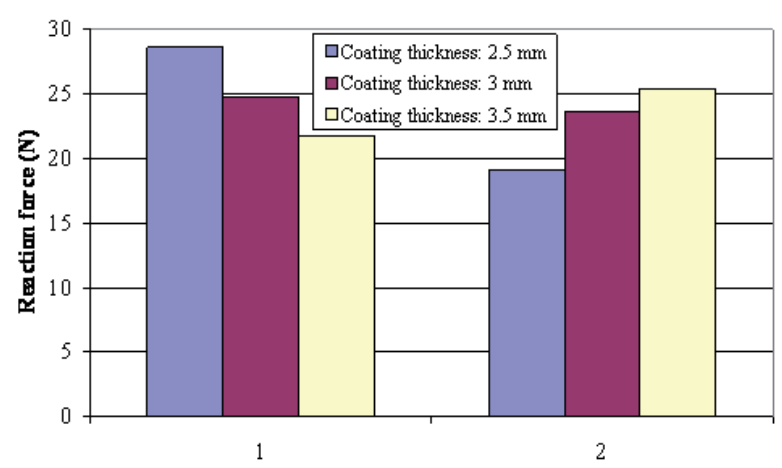

Fig. 15. Reaction force applied to the coating bottom within the range: (1) $x \in[0,1.6]-(2) x \in[1.6,2.54]$.

found while it was expected that hardness should be always decreasing with thickness. By focussing the study on the central part of the coating, which is the most significant region to account for plastic effects, we have demonstrated that this minimum hardness is produced by the boundary conditions influence on hardness measurement.

The good agreement found between numerical and experimental results demonstrates that a bilinear hardening model is sufficient to simulate the loading cycle of the HR15Y hardness test applied to the abradable layers studied in this work. This simple model may be proposed as a starting assumption for the simulation of other experiments, to be validated afterwards. However, in order to investigate more accurately a widest range of materials and loading conditions, the proposed method could be easily extended to multilinear plastic materials. To include such materials in the identification process, the number of design variables used for the optimization stage should be increased and a multilinear plastic law used rather than a bilinear one for the finite element analysis.

\section{Acknowledgements}

This research work was completed from May 2002 to May 2006 within the European Seal-Coat project funded by the European Commission under the FP5 Growth Program. The Seal-Coat project has involved UTBM (Peter Chandler, in charge of the Project Management, and the authors of this paper) and the following partners:

ESIL, Dublin/IE (Barry O'Reilly)

Rolls-Royce plc, Derby/GB (Noel Hopkins and Chris Sellars) MTU Aero Engines GmbH, München/DE (Manuel Hertter and Ulrike Hain)

Euromat GmbH, Heinsberg/DE (Ulrich Morkramer) RWTH Aachen University, Aachen/DE (Jochen Zwick) Neomet Limited, Manchester/GB (Dieter Sporer) Institute of Plasma Physics, Praha/CZ (Jiri Matejicek) RALSA, Langreo/ES (Ramiro Rilla)

\section{References}

1. R.K. Schmid, F. Gharispoor, M. Dorfman, X. Wei, An Overview of Compressor Abradables, International Thermal Spray Conference 2000 (Montréal, 2000) pp. $1087-1093$

2. J. Mackerle, Finite Elements in Analysis and Design 37, $811(2001)$

3. Y.P. Cao, J. Lu, Acta Materialia 52, 4023 (2004)

4. J.L. He, S. Veprek, Surface and Coatings Technology 163164, 374 (2003)

5. X. Cai, H. Bangert, Thin Solid Films 264, 59 (1995)

6. A. Nayebi, O. Bartier, G. Mauvoisin, R. El Abdi, Internat. J. Mech. Sci. 43, 2679 (2001)

7. I.H. Choi, C.H. Lim, Composite Structures 66, 125 (2004)

8. J.M. Olaf, E. Sommer, Finite-element analysis of indentation experiments in surface-coated materials, Experimental mechanics (1993), pp. 201-204

9. M. Zhao, N. Ogasawara, N. Chiba, X. Chen, Acta Materialia 54, Issue 1, 23 (2006)

10. H. Lee, J.H. Lee, G.M. Pharr, J. Mech. Phys. Solids 53, 2037 (2005)

11. K.C. Tang, R.D. Arnell, Thin Solid Films 355-356, 263 (1999)

12. S. Kucharski, Z. Mroz, Materials Sci. Eng. A318, 65 (2001)

13. M. Beghini, L. Bertini, V. Fontanari, Internat. J. Solids Struct. 43, 2441 (2006)

14. K.D. Bouzakis, N. Michailidis, G. Erkens, Surface and Coatings Technology 142102 (2001)

15. ANSYS HTML Online Documentation, Release 8.1, 2004

16. S. Swaddiwudhipong, K.K. Tho, Z.S. Liu, K. Zeng, Internat. J. Solids Struct. 42, 69 (2005)

17. ASTM International, Standard Test Methods for Rockwell Hardness and Rockwell Superficial Hardness of Metallic Materials, E18-05e1, 2005

18. H.I. Faraoun, J.L. Seichepine, C. Coddet, H. Aourag, J. Zwick, N. Hopkins, D. Sporer, M. Hertter, 2006, Surface and Coatings Technology 200, Issues 22-23, 6578 (2006)

19. L. Ma, S.R. Low, J. Song, Journal of Testing and Evaluation 31, 514 (2003)

20. L. Gan, B. Ben-Nissan, Computational Materials Science 8, 273 (1997)

21. M. Zhao, N. Ogasawara, N. Chiba, X. Chen, Acta Materialia 54, Issue 1, 23 (2006)

22. N. Ogasawara, N. Chiba, X. Chen, Scripta Materialia 54, 65 (2006)

23. K.K. Tho, S. Swaddiwudhipong, Z.S. Liu, K. Zeng, Materials Sci. Eng. A 390, 202 (2005)

24. Y.-T. Cheng, C.-M. Cheng, Mat. Sci. Eng.: R: Reports 44, 91 (2004)

25. D.-C. Lim, G.-C. Chen, S.-B. Lee, J.-H. Boo, Surface and Coating Technology 163-164, 318 (2003)

26. N. Ye, K. Komvopoulos, Journal of Tribology, 125, 685 (2003) 